\title{
Learning a Structure Adaptive Dictionary for Sparse Representation based Classification
}

\author{
Heyou Chang ${ }^{1}$, Meng Yang ${ }^{2}$ and Jian Yang ${ }^{1}$ \\ ${ }^{1}$ School of Computer Science and Engineering, Nanjing University of Science and \\ Technology, Nanjing, P.R. China \\ 2 Shenzhen University, Shenzhen, China
}

\begin{abstract}
Dictionary learning (DL), playing a key role in the success of sparse representation, has led to state-of-the-art results in image classification tasks. Among the existing supervised dictionary learning methods, the label of each dictionary atom is predefined and fixed, i.e., each dictionary atom is either associated to all classes or assigned to a single class. In this paper, we propose a structure adaptive dictionary learning (SADL) method to learn the relationship between dictionary atoms and classes, which is indicated by a binary association matrix and jointly optimized with the dictionary. The binary association matrix can not only represent class-specific dictionary atoms, but also hyper-class dictionary atoms shared by multiple classes. Furthermore, discrimination is explored by introducing Fisher criterion on coding coefficient and reducing between-class dictionary coherence. The extensive experimental results have shown the proposed SADL can achieve better performance than previous supervised dictionary learning methods on various classification databases.
\end{abstract}

Keywords: Structure Adaptive Dictionary Learning, Sparse Representation, Fisher Criterion, Image Classification

\section{Introduction}

Sparse representation has been shown to be a powerful and efficient tool for various vision tasks [1, 2, 3]. The basic model of sparse representation suggests that natural signals can be compactly expressed or efficiently approximated as a linear combination of dictionary atoms, where the coefficients 
are sparse. The dictionary plays an important role in the success of sparse representation [4]. Using off-the-shelf bases as dictionary (wavelets) might be universal to all types of images but would not be effective enough for some specific image classification tasks (such as face classification). Learning a dictionary from training samples has led to the state-of-the-art results in many particular applications, such as image processing [5], face recognition [6, 7] and image classification [8, 9].

Current prevailing supervised DL methods can be divided into three main categories. In the first category, a dictionary shared by all classes and a classifier trained on the representation coefficients computed on the dictionary are learnt jointly [5, 6, 10, 11, 12, 13. Based on [6], Song et al [14] adopted sparse error correction (SEC) model to minimize the energy of the errors and proposed SEC-DKSVD for iris recognition. By introducing Fisher criterion, Zheng et al [15] proposed Fisher discriminative KSVD (FD-KSVD) to learn an over complete discriminative dictionary and an optimal linear classifier for image classification and Dong et al [16] proposed a supervised dictionary learning algorithm for action recognition in still images. Methods in the second category try to learn a dictionary whose atoms have correspondence to the class labels. Sprechmann et al [17] learnt a class-specific dictionary with sparse coefficients and applied it to signal clustering. Ramirez et al [18] introduced an incoherence term between the dictionaries associated with different classes, which encourages the dictionaries belonged different classes to be as independent as possible. Based on [18], Wang et al [19] proposed a class-specific DL method for sparse modeling in action recognition. Yang et al [20] introduced Fisher discrimination both in the sparse coding coefficients and class-specific representations. Castrodad et al [21] learnt a set of actionspecific dictionaries with non-negative penalty on both dictionary atoms and representation coefficients. Instead of adding the sparsity constraint on the coefficients of each input signal, Meng et al [22] proposed to learn a dictionary under global sparsity constraint to fittingly assign the atoms of the dictionary to represent signals. In the third category, a hybrid dictionary which contains a set of class-specific dictionaries and a shared dictionary were learnt. Kong et al [8] learnt a hybrid dictionary by introducing an incoherence penalty term to the class-specific sub-dictionaries. Zhou et al [23] learnt a hybrid dictionary with a Fisher-like regularization on the coding coefficients.

Although these DL methods have achieved good performance, the label and the property of dictionary atom are pre-given, which may not be able to reflect the practical data structure accurately, i.e., particularity and com- 


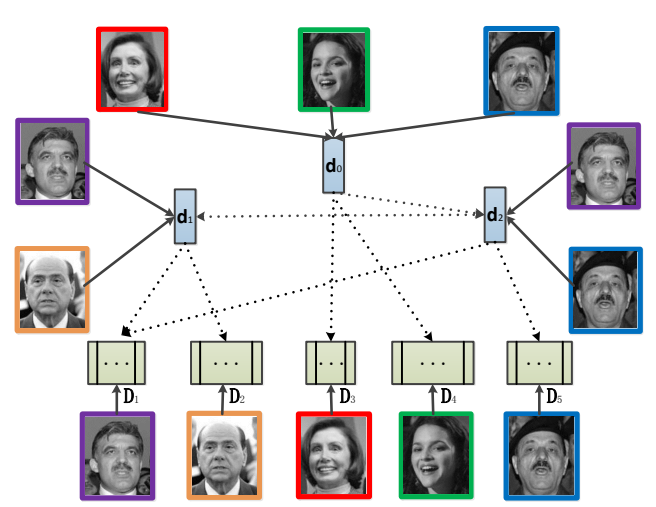

(a)

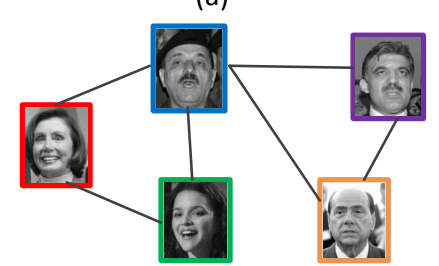

(b)

Figure 1: (a) The relationships between 5 persons and dictionary atoms learnt on LFW database. (b) The graph structure between class labels.

monality shared by multiple classes. In addition, how to balance the size of each class-specific part in the dictionary is not a trivial task. Yang et al 24] proposed a latent dictionary learning (LDL) method to build the relationship between dictionary atoms and class labels by learning a real-number latent matrix. Although the promising performance has been reported, the real-number latent matrix learnt by LDL could not represent which classes a dictionary atom belongs to. The label and the property of each dictionary atom were still blurry.

In this paper, we propose to learn a dictionary where the label and the property of each dictionary atom can be learnt adaptively. Although highorder group structure among data has been explored by building a probabilistic model in [25, 26], how to define the relationship between class labels and dictionary atoms is seldom discussed. In order to avoid predefining the relationships between dictionary atoms and class labels, we proposed to learn a structure adaptive dictionary with a binary matrix to indicate the relationships. As shown in Fig,1(a), dictionary atom $\mathbf{d}_{0}, \mathbf{d}_{1}$ and $\mathbf{d}_{2}$ are shared by more than one class, and $\mathbf{D}_{1}, \cdots, \mathbf{D}_{5}$ are class-specific sub- 
dictionary. In traditional dictionary learning methods, the shared atoms (e.g., $\mathbf{d}_{0}, \mathbf{d}_{1}$ and $\mathbf{d}_{2}$ ) are assumed to be shared by all classes. The size of each sub-dictionary (shared sub-dictionary and class-specific sub-dictionary) is also predefined. In SADL, it is easy to see that the shared dictionary atom $\mathbf{d}_{0}$ is shared by 3 persons; $\mathbf{d}_{1}$ and $\mathbf{d}_{2}$ are shared by 2 persons respectively. At the same time, $\mathbf{d}_{1}$ and $\mathbf{d}_{2}, \mathbf{d}_{0}$ and $\mathbf{d}_{2}$ are shared by one person respectively (violet box and turquoise box respectively). Besides, the size of each class-specific sub-dictionary $\mathbf{D}_{1}, \cdots, \mathbf{D}_{5}$ and shared sub-dictionary $\left[\mathbf{d}_{0}, \mathbf{d}_{1}, \mathbf{d}_{2}\right]$ can be adaptively learned during dictionary learning. Compared to LDL, the binary relationship is more meaningful because it clearly shows which class or classes a dictionary atom is associated with. On the other hand, to enhance the discrimination of the dictionary, a discrimination ter$\mathrm{m}$ based on Fisher discrimination criterion is applied on the coefficients. A weighted dictionary coherence term is also added to reduce the correlation of dictionary atoms between different classes. Compared with other supervised DL methods, SADL has two advantages. Firstly, SADL can adaptively adjust the label and property of each dictionary atom, which results in a more compact dictionary. Secondly, SADL can construct a graph structure between class labels by exploring the class relation among dictionary atoms, shown as Fig.1(b). Extensive experiments on various image classification tasks, such as face recognition, gender classification and action recognition, showed the proposed SADL can achieve competitive performance with those state-of-the-art dictionary learning methods.

The reminder of this paper is organized as follows: Related supervised dictionary learning methods are introduced in Section 2. Then in Section 3 we present the proposed structure adaptive dictionary learning method. In Section 4, we evaluate the performance of SADL on various image classification tasks. Finally, we conclude this paper in Section 5.

\section{Related Work}

\subsection{All-class-shared Dictionary Learning}

In all-class-shared dictionary learning, a dictionary shared by all classes is learnt. In order to improve the classification ability, it is popular to learn a shared dictionary and a classifier on the coefficients jointly. Marial et al [10] proposed to learn a discriminative dictionary with a logistic function simultaneously. Pham et al [1] proposed to learn a dictionary with linear classifier for both the labeled and unlabeled data. Inspired by the work 
of [11], Zhang et al [6] proposed discriminative KSVD (DKSVD) for face recognition. Jiang et al [13] proposed Label-Consistent KSVD (LCKSVD) by adding a label consistency regularization. The optimization problem can be written as:

$$
\min _{\mathbf{D}, \mathbf{Z}} \sum_{i=1}^{C}\left(\left\|\mathbf{X}_{i}-\mathbf{D} \mathbf{Z}_{i}\right\|_{F}^{2}\right)+f_{\mathbf{Z}}(\mathbf{Z}) \quad \text { s.t. }\left\|\mathbf{d}_{m}\right\|_{2}^{2}=1 \quad \forall m
$$

where $\mathbf{D}=\left[\mathbf{d}_{1}, \mathbf{d}_{2}, \ldots, \mathbf{d}_{K}\right] \in \mathcal{R}^{d \times K}, f_{\mathbf{Z}}(\mathbf{Z})$ is a function on $\mathbf{Z}$.

\subsection{Class-specific Dictionary Learning}

In class-specific dictionary learning, the atoms in the learnt dictionary $\mathbf{D}=\left[\mathbf{D}_{1}, \ldots, \mathbf{D}_{C}\right]$ have class labels correspond to the subject classes, where $\mathbf{D}_{i} \in \mathcal{R}^{d \times K_{i}}$ is the sub-dictionary corresponding to class $i$. Sprechmann et al [17] learnt a dictionary for each class and applied it to signal clustering. Ramirez et al [18] introduced an incoherence term between the dictionaries associated with different classes, which encourages the dictionaries belonged to different classes to be independent as much as possible. Based on [18, Wang et al [19] proposed a class-specific dictionary learning method for sparse modeling in action recognition. Yang et al [20] introduced Fisher discrimination both in the sparse coding coefficients and class-specific representations to make both the representation residual and the representation coefficients be discriminative. The dictionary $\mathbf{D}$ can be learnt class by class:

$$
\min _{\mathbf{D}, \mathbf{Z}} \sum_{i=1}^{C}\left(\left\|\mathbf{X}_{i}-\mathbf{D}_{i} \mathbf{Z}_{i}\right\|_{F}^{2}\right)+f_{\mathbf{Z}}(\mathbf{Z})+f_{\mathbf{D}}(\mathbf{D}) \quad \text { s.t. }\left\|\mathbf{d}_{m}\right\|_{2}^{2}=1 \quad \forall m
$$

where $\mathbf{D}=\left[\mathbf{D}_{1}, \mathbf{D}_{2}, \ldots, \mathbf{D}_{C}\right] \in \mathcal{R}^{d \times K}, \mathbf{D}_{i}=\left[\mathbf{d}_{1}^{i}, \ldots, \mathbf{d}_{K_{i}}^{i}\right], K=\sum_{i=1}^{C} K_{i}, f_{\mathbf{Z}}(\mathbf{Z})$ and $f_{\mathbf{D}}(\mathbf{D})$ are functions on $\mathbf{Z}$ and $\mathbf{D}$, respectively.

\subsection{Hybrid Dictionary Learning}

In hybrid dictionary learning, a shared dictionary $\mathbf{D}_{C+1}$ by all classes and a set of class-specific dictionaries $\mathbf{D}_{i}, i \in\{1, . ., C\}$ were learnt. Kong et al [8] learnt a hybrid dictionary by introducing an incoherence penalty term to the class-specific sub-dictionaries. Zhou et al [23] learnt a hybrid dictionary with a Fisher-like regularization on the coding coefficients. The optimization problem can be written as:

$$
\min _{\mathbf{D}, \mathbf{Z}} \sum_{i=1}^{C}\left(\left\|\mathbf{X}_{i}-\left[\mathbf{D}_{i}, \mathbf{D}_{C+1}\right] \mathbf{Z}_{i}\right\|_{F}^{2}\right)+f_{\mathbf{Z}}(\mathbf{Z})+f_{\mathbf{D}}(\mathbf{D}) \quad \text { s.t. }\left\|\mathbf{d}_{m}\right\|_{2}^{2}=1 \quad \forall m
$$


where $\mathbf{D}=\left[\mathbf{D}_{1}, \mathbf{D}_{2}, \ldots, \mathbf{D}_{C}, \mathbf{D}_{C+1}\right] \in \mathcal{R}^{d \times K}, K=\sum_{i=1}^{C+1} K_{i}$

\section{The Proposed Method}

In this section, we first present SADL method, and then describe the optimization procedure of SADL. The time complexity and classification scheme are also presented at the end of this section.

\section{1. $S A D L$}

Suppose that there are $C$ classes and $\mathbf{X}=\left[\mathbf{X}_{1}, \mathbf{X}_{2}, \cdots, \mathbf{X}_{C}\right] \in \mathcal{R}^{d \times N}$ is the set of training samples, where $\mathbf{X}_{i} \in \mathcal{R}^{d \times N_{i}}\left(N=\sum_{i=1}^{C} N_{i}\right)$ represents the data from class $i, N_{i}$ is the number of the training samples from class $i$.

The proposed SADL is defined as:

$$
\begin{aligned}
& \min _{\mathbf{D}, \mathbf{Z}, \mathbf{W}} \sum_{i=1}^{C}\left(\left\|\mathbf{X}_{i}-\mathbf{D} \operatorname{diag}\left(\mathbf{w}_{i}\right) \mathbf{Z}_{i}\right\|_{F}^{2}+\gamma_{1}\left\|\mathbf{Z}_{i}\right\|_{1}\right)+\gamma_{2} f_{\mathbf{Z}}(\mathbf{Z})+\gamma_{3} f_{\mathbf{D}}(\mathbf{D}) \\
& \text { s.t. } \mathbf{w}_{i} \in\{0,1\}^{K \times 1} \forall i, \quad\left\|\mathbf{d}_{m}\right\|_{2}^{2}=1 \forall m
\end{aligned}
$$

where $\mathbf{D} \in \mathcal{R}^{d \times K}$ is the overall dictionary, $\mathbf{Z}_{i} \in \mathcal{R}^{K \times N_{i}}$ is coefficients of $\mathbf{X}_{i}$ over $\mathbf{D}$, and $\operatorname{diag}\left(\mathbf{w}_{i}\right)$ is a diagonal matrix with $\mathbf{w}_{i}$ as its diagonal vector. $\mathbf{W}=\left[\mathbf{w}_{1}, \ldots, \mathbf{w}_{C}\right] \in\{0,1\}^{K \times C}$ is a binary matrix. $f_{\mathbf{Z}}(\mathbf{Z})=\operatorname{tr}\left(S_{W}(\mathbf{Z})-\right.$ $\left.S_{B}(\mathbf{Z})\right)+\|\mathbf{Z}\|_{F}^{2}$ is a discrimination term imposed on the coefficient matrix $\mathbf{Z}$, where $\operatorname{tr}(\cdot)$ is the trace opreator. The term $\|\mathbf{Z}\|_{F}^{2}$ could make $f_{\mathbf{Z}}(\cdot)$ smoother and convex [9]. $f_{\mathbf{D}}(\mathbf{D})=\sum_{m=1}^{K} \sum_{n \neq m}\left(\mathbf{d}_{m}^{T} \mathbf{d}_{n}\right)^{2} \sum_{i=1}^{C} \sum_{j \neq i} w_{m, i} w_{n, l}$ is an incoherence term on the dictionary items. $\gamma_{1}, \gamma_{2}$ and $\gamma_{3}$ are scalar parameters.

Methods in the all-class-shared dictionary learning require that the learnt dictionary $\mathbf{D}$ should represent all training samples of every class $i$ well, i.e. $\mathbf{X}_{i} \approx \mathbf{D Z}_{i}=\mathbf{D} \operatorname{diag}\left(\mathbf{w}_{i}\right) \mathbf{Z}_{i}$, where

$$
\mathbf{w}_{i}=[\underbrace{1,1, \cdots, 1}_{K}]^{T} .
$$

Methods in the class-specific dictionary learning try to learn a classspecific dictionary $\mathbf{D}=\left[\mathbf{D}_{1}, \mathbf{D}_{2}, \cdots, \mathbf{D}_{C}\right] \in \mathcal{R}^{d \times K}$ and coefficients $\mathbf{Z}=$ $\left[\mathbf{Z}_{1}, \mathbf{Z}_{2}, \cdots, \mathbf{Z}_{C}\right] \in \mathcal{R}^{K \times N}$, where $\mathbf{D}_{i} \in \mathcal{R}^{d \times K_{i}}\left(K=\sum_{i=1}^{C} K_{i}\right)$ stands for the sub-dictionary associated with class $i, \mathbf{Z}_{i}$ is the coding coefficients of $\mathbf{X}_{i}$ 
over $\mathbf{D}$. The samples of $i$-th class should be well reconstructed by $\mathbf{D}_{i}$, i.e. $\mathbf{X}_{i} \approx \mathbf{D}_{i} \mathbf{Z}_{i}^{i}=\mathbf{D} \operatorname{diag}\left(\mathbf{w}_{i}\right) \mathbf{Z}_{i}$, where

$$
\mathbf{w}_{i}=[\underbrace{0, \cdots, 0}_{\sum_{j=1}^{i-1} K_{j}}, \underbrace{1, \cdots, 1}_{K_{i}}, \underbrace{0, \cdots, 0}_{\sum_{j=i+1}^{C} K_{j}}]^{T}
$$

$\mathbf{Z}_{i}=\left[\mathbf{Z}_{i}^{1} ; \mathbf{Z}_{i}^{2} ; \cdots ; \mathbf{Z}_{i}^{C}\right] \in \mathcal{R}^{K_{i} \times N_{i}}, \mathbf{Z}_{i}^{j}$ is the sub-matrix containing the coding coefficients of $\mathbf{X}_{i}$ over $\mathbf{D}_{j}$.

In the hybrid dictionary learning methods, samples from $i$-th class should be represented by $\mathbf{D}_{i}$ and the shared dictionary $\mathbf{D}_{s} \in \mathcal{R}^{m \times K_{s}}$, i.e. $\mathbf{X}_{i} \approx$ $\left[\mathbf{D}_{i}, \mathbf{D}_{C+1}\right]\left[\mathbf{Z}_{i}^{i} ; \mathbf{Z}_{i}^{C+1}\right]=\mathbf{D} \operatorname{diag}\left(\mathbf{w}_{i}\right) \mathbf{Z}_{i}$, where

$$
\mathbf{w}_{i}=[\underbrace{0, \cdots, 0}_{\sum_{j=1}^{i-1} K_{j}}, \underbrace{1, \cdots, 1}_{K_{i}}, \underbrace{0, \cdots, 0}_{\sum_{j=i+1}^{C} K_{j}}, \underbrace{1, \cdots, 1}_{K_{C+1}}]^{T}
$$

The size of sub-dictionary and the relationships between dictionary atoms and class labels in the methods mentioned above are predefined and fixed, which may be not accurate for practical data. In SADL, the relationships which are indicated by a binary matrix $\mathbf{W}$ is updated with the updating of dictionary $\mathbf{D}$ and coefficients $\mathbf{Z}$. $\mathbf{W}$ is adaptively updated so that it can assign dictionary atoms to classes more flexibly. It is clear to see that the all-class-shared, class-specific, and hybrid dictionary learning methods can be viewed as specific cases of SADL.

In the objective function of SADL, the first term ensures that the learnt sub-dictionary $\mathbf{D}_{i}$ from $\mathbf{X}_{i}$ is adapted for representation of the data and that the coding coefficient is sparse. The second term is the Fisher criterion which increases the discrimination capability of $\mathbf{Z}$ by minimizing the withinclass scatter of $\mathbf{Z}$, denoted by $S_{W}(\mathbf{Z})$, and by maximizing the between-class scatter of $\mathbf{Z}$, denoted by $S_{B}(\mathbf{Z})$. The smaller the within-class scatter and the bigger the between-class scatter, the more accurate the $\mathrm{W}$. The third term is applied to reduce the correlations between dictionary atoms associated to different classes. If dictionary atom $\mathbf{d}_{m}$ and $\mathbf{d}_{n}$ are correlated, $\mathbf{d}_{m}^{T} \mathbf{d}_{n}$ is big. By minimizing the third term, the $\sum_{i=1}^{C} \sum_{j \neq i} w_{m, i} w_{n, j}$ will be as small as possible, which $\mathbf{d}_{m}$ and $\mathbf{d}_{n}$ would be very likely to have the same class labels.

\subsection{Optimization of $S A D L$}

The proposed objective function in Eq.(4) is solved by alternatively optimizing the following two sub-problems:

1) Fixing $\mathbf{W}$, update $\mathbf{Z}$ and $\mathbf{D}$.

2) Fixing $\mathbf{Z}$ and $\mathbf{D}$, update $\mathbf{W}$. 


\subsubsection{Update $\mathbf{Z}$ and $\mathbf{D}$}

When $\mathbf{W}$ is fixed, the problem (4) can be rewritten as:

$$
\begin{aligned}
& \min _{\mathbf{D}, \mathbf{Z}} \sum_{i=1}^{C}\left(\left\|\mathbf{X}_{i}-\mathbf{D}\left(\operatorname{diag}\left(\mathbf{w}_{i}\right)\right) \mathbf{Z}_{i}\right\|_{F}^{2}+\gamma_{1}\left\|\mathbf{Z}_{i}\right\|_{1}\right) \\
& \quad+\gamma_{2}\left(\operatorname{tr}\left(S_{W}(\mathbf{Z})-S_{B}(\mathbf{Z})\right)+\|\mathbf{Z}\|_{F}^{2}\right) \\
& \quad+\gamma_{3} \sum_{m=1}^{K} \sum_{n \neq m}\left(\mathbf{d}_{m}^{T} \mathbf{d}_{n}\right)^{2} \Theta_{m, n} ; \\
& \text { s.t. }\left\|\mathbf{d}_{m}\right\|_{2}^{2}=1 \quad \forall m
\end{aligned}
$$

where $\Theta_{m, n}=\sum_{i=1}^{C} \sum_{j \neq i} w_{m, i} w_{n, j}$. The problem (5) can be optimized by alternatively solving $\mathbf{Z}$ and $\mathbf{D}$, and it is convex with either of them with the other one fixed.

First, $\mathbf{D}$ is assumed to be fixed, the coefficients $\mathbf{Z}_{i}$ can be updated class by class and all $\mathbf{Z}_{j}(j \neq i)$ are kept fixed. Then, the $\mathbf{Z}_{i}$ can be updated by solving following optimization:

$$
\begin{aligned}
\left.\min _{\mathbf{Z}_{i}}\left\|\mathbf{X}_{i}-\mathbf{D}\left(\operatorname{diag}\left(\mathbf{w}_{i}\right)\right) \mathbf{Z}_{i}\right\|_{F}^{2}+\gamma_{1}\left\|\mathbf{Z}_{i}\right\|_{1}\right) \\
\quad+\gamma_{2}\left(\left\|\mathbf{Z}_{i}-\mathbf{M}_{i}\right\|_{F}^{2}-\sum_{j=1}^{C}\left\|\mathbf{M}_{j}-\mathbf{M}\right\|_{F}^{2}+\left\|\mathbf{Z}_{\mathbf{i}}\right\|_{F}^{2}\right)
\end{aligned}
$$

where $\mathbf{M}_{j}$ and $\mathbf{M}$ are the mean vector matrices (by taking $N_{j}$ mean vector $\mathbf{m}_{j}$ or $\mathbf{m}$ as its column vectors) of class $j$ and all classes respectively. It can be solved by using Iterative Projection Method [27, 20].

Next, after $\mathbf{Z}$ is solved, $\mathbf{D}$ can be updated atom by atom. Letting $\mathbf{Y}=$ $\left[\mathbf{Y}_{1}, \mathbf{Y}_{2}, \ldots, \mathbf{Y}_{C}\right]$, where $\mathbf{Y}_{i}=\operatorname{diag}\left(\mathbf{W}_{i}\right) \mathbf{Z}_{i}$, the objective function in Eq.(5) then becomes to:

$$
\min _{\mathbf{D}}\|\mathbf{X}-\mathbf{D Y}\|_{F}^{2}+\gamma_{3} \sum_{m=1}^{K} \sum_{n \neq m}\left(\mathbf{d}_{m}^{T} \mathbf{d}_{n}\right)^{2} \Theta_{m, n} \quad \text { s.t. }\left\|\mathbf{d}_{m}\right\|_{2}^{2}=1 \quad \forall m
$$

For the updating of $n$-th dictionary atom, the above object function will be converted into:

$$
\min _{\mathbf{d}_{n}} \operatorname{Tr}\left(\mathbf{D}^{\mathbf{T}} \mathbf{D} \boldsymbol{\Gamma}-\mathbf{2} \mathbf{D}^{\mathbf{T}} \boldsymbol{\Lambda}\right)+\gamma_{3} \sum_{n \neq m}\left(\mathbf{d}_{m}^{T} \mathbf{d}_{n}\right)^{2} \Theta_{m, n} \quad \text { s.t. }\left\|\mathbf{d}_{n}\right\|_{2}^{2}=1
$$


where $\boldsymbol{\Gamma}=\mathbf{Y} \mathbf{Y}^{T}, \boldsymbol{\Lambda}=\mathbf{X} \mathbf{Y}^{T}$. This is also convex for $\mathbf{d}_{n}$. Based on the dictionary updating algorithm of [28], $\mathbf{d}_{n}$ can be updated by:

$$
\begin{aligned}
& \mathbf{v}=\left(\boldsymbol{\Gamma}_{n, n} \mathbf{I}+2 \gamma_{3} \mathbf{I}_{p}\right)^{-1}\left(\boldsymbol{\Lambda}_{n}-\mathbf{D} \boldsymbol{\Gamma}_{n}-2 \gamma_{3} \mathbf{P} \mathbf{D}_{n}\right) \\
& \mathbf{d}_{n}=\mathbf{v} /\|\mathbf{v}\|_{2}
\end{aligned}
$$

where $\mathbf{P}=\sum_{n \neq m} \mathbf{d}_{m} \mathbf{d}_{m}^{T} \Theta_{m, n}, \mathbf{I}$ is an identity, $\mathbf{I}_{p}$ is a diagonal matrix with the same diagonal elements as $\mathbf{P}, \boldsymbol{\Gamma}_{n, n}$ is the element in $n$-th row and $n$-th column of $\boldsymbol{\Gamma}$, and $\boldsymbol{\Lambda}_{n}, \boldsymbol{\Gamma}_{n}, \mathbf{D}_{n}$ are the $n$-th column vectors of $\boldsymbol{\Lambda}, \boldsymbol{\Gamma}, \mathbf{D}$, respectively.

\subsubsection{Update $\mathbf{W}$}

When $\mathbf{D}$ and $\mathbf{Z}$ are fixed, we can update the binary matrix $\mathbf{W}$ column by column. For updating $\mathbf{w}_{i}$ for $i$-th class, the problem (4) can be rewritten as:

$$
\begin{aligned}
& \min _{\mathbf{w}_{i}}\left\|\mathbf{X}_{i}-\mathbf{D}\left(\operatorname{diag}\left(\mathbf{w}_{i}\right)\right) \mathbf{Z}_{i}\right\|_{F}^{2} \\
& +\gamma_{3} \sum_{m=1}^{K} w_{m, i} \sum_{n \neq m}\left(\mathbf{d}_{m}^{T} \mathbf{d}_{n}\right)^{2} \sum_{j \neq i}^{C} w_{n, j} \\
& \text { s.t. } \mathbf{w}_{i} \in\{0,1\}^{K \times 1} \quad \forall i
\end{aligned}
$$

Since $\mathbf{w}_{i} \in\{0,1\}^{K \times 1}$, a greedy optimization method is applied to get the binary vector $\mathbf{w}_{i}$. We first set all the elements in $\mathbf{w}_{i}$ as 0 , then we search for the single best dictionary atom that can minimize Eq.(11) and set the corresponding element in $\mathbf{w}_{i}$ to 1 . Afterwards, based on the previously chosen dictionary atoms, we search for next atom that can further minimize Eq. (11). After several rounds of searching, when the values of the objective function in Eq. 111) in adjacent iterations are close enough or the maximum number of iterations is reached, we stop updating $\mathbf{w}_{i}$. The algorithm of updating $\mathbf{W}$ is summarized in Algorithm 1.

\subsection{Time Complexity and Convergence}

In the proposed SADL algorithm, the time complexity of updating the coding coefficients is $\sum_{i} N_{i} O\left(d^{2} K_{i}^{\varepsilon}\right)$ [9], where $\varepsilon \geq 1.2$ is a constant, $d$ is the feature dimensionality, $N_{i}$ is the number of training samples in class $i, K_{i}$ is the number of dictionary atoms in $\mathbf{D}_{i}$. The time complexity of updating dictionary atoms is $O\left(K d^{2}\right)$, where $K=\sum_{i=1}^{C} K_{i}$. The time complexity of updating $\mathbf{W}$ is $\sum_{i} O\left(K d+N_{i} d\right)$. In our experiments, the main iteration number is less than 5 , and $\mathbf{W}$ is updated after every 5 updates of $\mathbf{D}$ and $\mathbf{Z}$. 

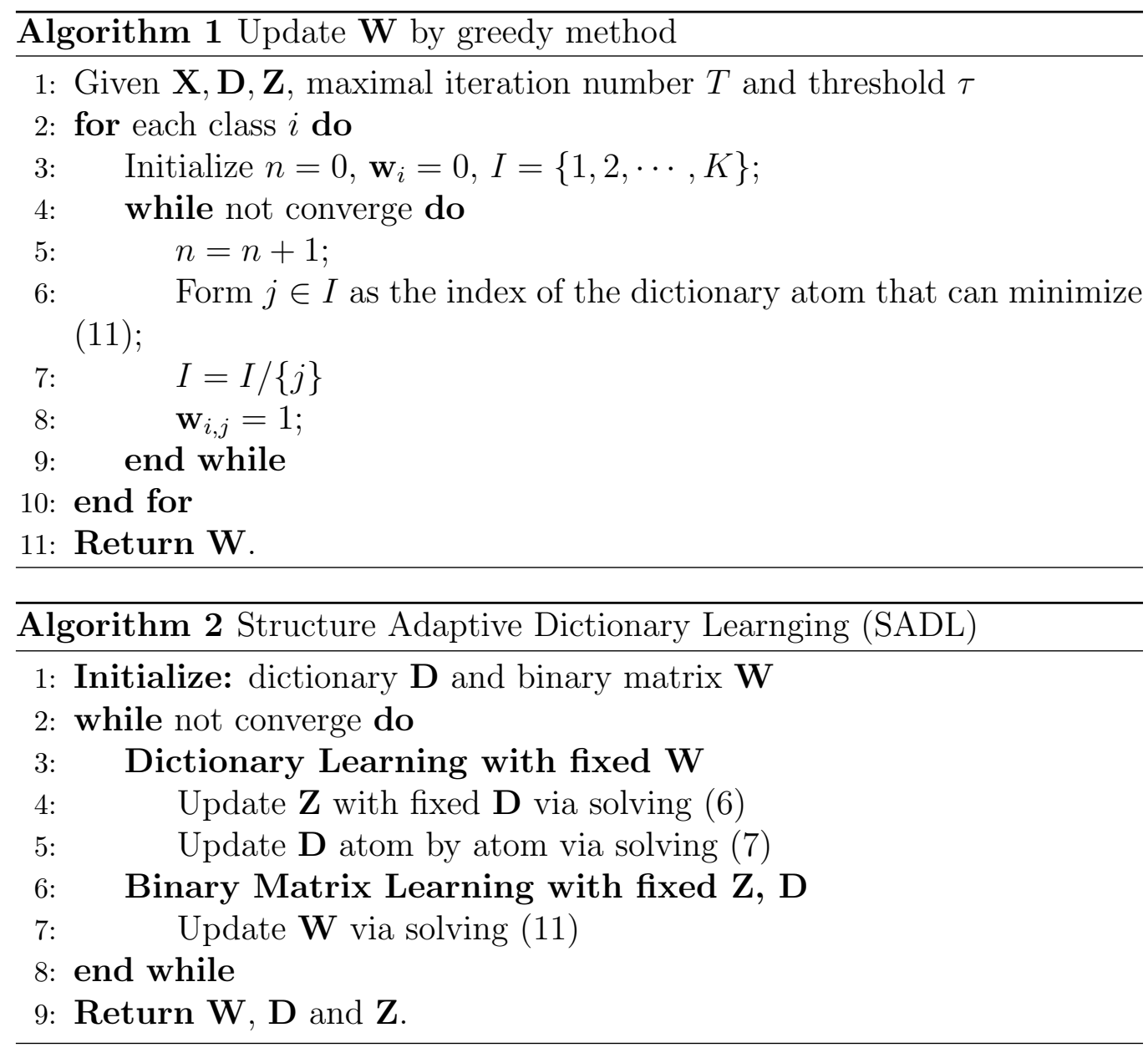

The whole algorithm of the proposed structure adaptive dictionary learning is summarized in Algorithm 2. The objective of SADL is lower-bounded. Since the objective of SADL decreases in both dictionary updating and binary matrix updating, the proposed algorithm can converges. The objective function value versus the iteration number on different databases are shown as Fig.2.

\subsection{Classification Scheme}

Once $\mathbf{D}$ and $\mathbf{W}$ are learnt, they could be used to present a query sample y and judge its label. With the Fisher criterion, both the representation residual and the representation coefficients will be discriminative, and we make use of both of them to achieve more accurate classification results. 

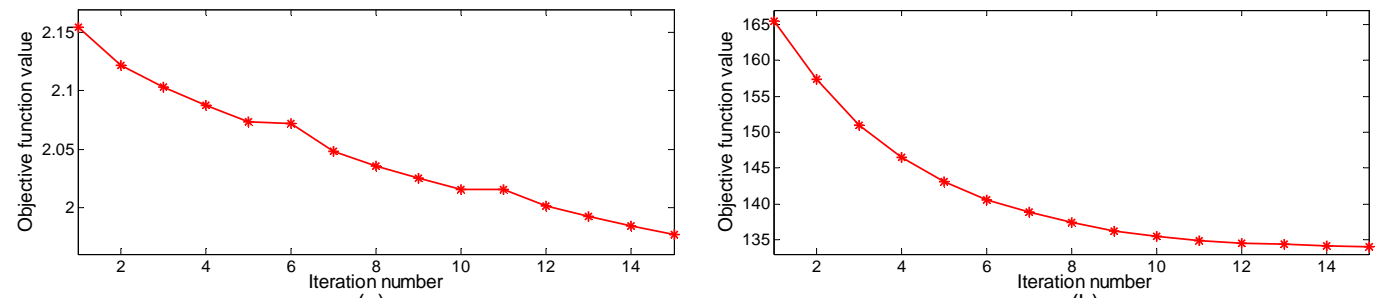

(a)

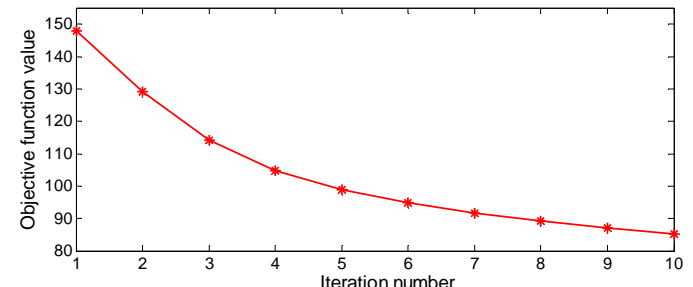

(c)

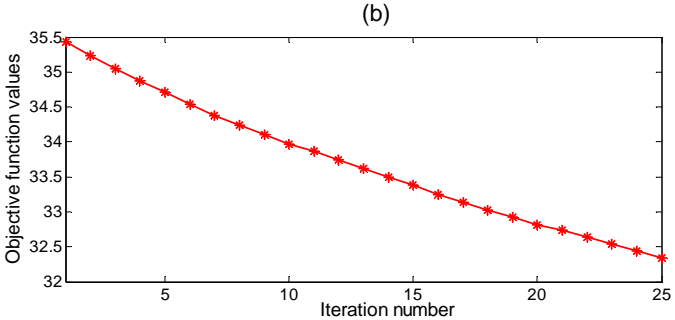

(d)

Figure 2: The objective function value versus the iteration number on different databases. (a) UCFSports, (b) ARGender with 25 dictionary atoms initialized per class, (c) ARGender with 250 dictionary atoms initialized per class, (d) LFWa.

According to the number of training samples per class, here we propose two classifiers: the global classifier (GC) for insufficient training samples situation and the local classifier (LC) for sufficient training samples situation, which are described as follows.

GC: When the number of training samples per class is relatively small, the learnt sub-dictionary $\mathbf{D}_{i}$ may not be able to faithfully represent the query samples of this class, and we represent the query sample $\mathbf{y}$ over the whole dictionary $\mathbf{D}$, which is represented as:

$$
\hat{\mathbf{z}}=\operatorname{argmin}_{z}\|\mathbf{y}-\mathbf{D z}\|_{2}^{2}+\lambda\|\mathbf{z}\|_{1}
$$

where $\lambda$ is a constant. By considering the discrimination capability of both representation residual and representation vector, we could define the following metric for classification:

$$
e_{i}=\left\|\mathbf{y}-\mathbf{D} \operatorname{diag}\left(\mathbf{w}_{i}\right) \hat{\mathbf{z}}\right\|_{2}^{2}+\beta\left\|\hat{\mathbf{z}}-\mathbf{m}_{i}\right\|_{2}^{2}
$$

where $\mathbf{m}_{i}$ is the coefficient mean vector of class $i, \beta$ is a preset weight to balance the contribution of the two term to classification. Then the classification is conducted via identity $(\mathbf{y})=\operatorname{argmin}_{i}\left\{e_{i}\right\}$. 
LC: When the number of training samples of each class is relatively large, the sub-dictionary $\mathbf{D}_{i}$ is able to well span the subspace of class $i$. Then we can represent $\mathbf{y}$ locally over each sub-dictionary $\mathbf{D}_{i}$ instead of the whole dictionary $\mathbf{D}$. Since we have forced the representation vector $\mathbf{z}_{i}$ to be close to their mean, i.e., $\boldsymbol{\rho}_{i}$, in the test stage, we can also force the representation vector of query sample $\mathbf{y}$ over $\mathbf{D}_{i}$ to be close to $\boldsymbol{\rho}_{i}$ so that the representation process can be more informative. With the above considerations, the local representation model is defined as:

$$
\hat{\mathbf{z}}_{i}=\operatorname{argmin}_{z_{i}}\left\|\mathbf{y}-\mathbf{D}_{i} \mathbf{z}_{i}\right\|_{2}^{2}+\lambda_{1}\left\|\mathbf{z}_{i}\right\|_{1}+\lambda_{2}\left\|\mathbf{z}_{i}-\boldsymbol{\rho}_{i}\right\|_{2}^{2}
$$

where $\lambda_{1}$ and $\lambda_{2}$ are constants. The metric used for classification can be readily defined as:

$$
e_{i}=\left\|\mathbf{y}-\mathbf{D}_{i} \hat{\mathbf{z}}_{i}\right\|_{2}^{2}+\lambda_{1}\left\|\hat{\mathbf{z}}_{i}\right\|_{1}+\lambda_{2}\left\|\hat{\mathbf{z}}_{i}-\boldsymbol{\rho}_{i}\right\|_{2}^{2}
$$

The final classification rule is still identity $(\mathbf{y})=\operatorname{argmin}_{i}\left\{e_{i}\right\}$.

\section{Experiments and results}

In this section, we verify the performance of SADL on three classification tasks: face recognition, gender classification and action classification with several latest DL methods, such as DKSVD [6], LCKSVD [13], DLSI [18], FDDL [20], COPAR [8], JDL [23], LDL [24]. We also report sparse representation based classifier (SRC) [2] and linear support vector machine (SVM). There are three parameters: $\gamma_{1}, \gamma_{2}, \gamma_{3}$ in all experiments. Sparse regulation $\gamma_{1}$ and Fisher criterion regulation $\gamma_{2}$ are determined via cross-validation. Like [24], the parameter $\gamma_{3}$ which control the dictionary coherence is set to $0.01 \times N /(K \times(K-1))$, where $N$ is the number of all training samples and $K$ is the size of dictionary.

In our experiment, we report the classification results of $\mathrm{GC}$ and $\mathrm{LC}$ (SRC-LC(GC), LDL-LC(GC), SADL-LC(GC) for gender classification and SRC-GC(LC), LDL-GC(LC), SADL-GC(LC) for action classification and face recognition) on each database.

\subsection{The Number of Dictionary Atoms}

Dictionary size is an important factor in dictionary learning based classification. However, there are rules to effectively set dictionary size. Generally 


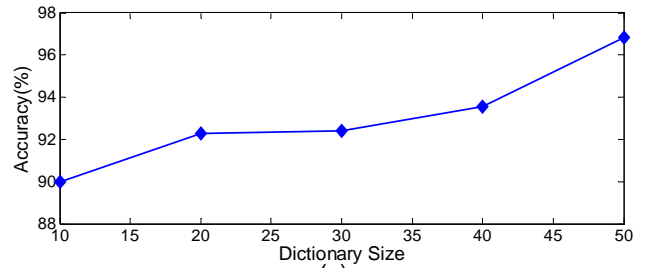

(a)

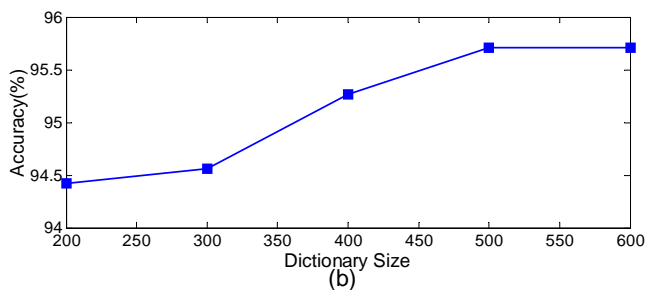

Figure 3: The accuracy versus initialized dictionary size on gender classification

speaking, dictionary size cannot be too small in order to have good representation ability. The classification accuracy will increases with the increase of dictionary size. When training samples are not sufficient, the size of dictionary size can be initially set as the number of training samples. When there is relatively sufficient training samples, the size of dictionary size should be smaller than the number training samples to reduce the redundancy.

As shown in Fig 3(a), we can see that when the initialized dictionary size is very small (e.g., from 10 to 50), the accuracy rises with the increase of dictionary size. When the initialized dictionary size is big, the accuracy will increases at first, and remains unchanged then with the increase of dictionary size, as Fig $3(\mathrm{~b})$. In the experiment, the proposed method could adaptively reduce the dictionary size to a proper size when the initialized dictionary size is big. For example, if the dictionary size is initialized as 500 or 600, the size of learnt dictionary reduces to 350 . This is the reason why the accuracy keeps unchanged when the initialized dictionary size is big.

\subsection{Face Recognition}

We evaluated SADL on the LFWa [29] face database for face classification. LFW is a large-scale database which contains variations of pose, illumination, expression, misalignment and occlusion, etc, as shown in Fig.4. We chose 4174 images in total from 143 persons with no less than 11 samples per person. For each person, the first 10 images were used as training data and the remaining images for testing. Just as the feature extraction in LDL, Histogram of Uniform-LBP was extracted via dividing a face image into $10 \times 8$ patches. Then we used PCA to reduce the histogram dimension to 1000. The classification accuracy is listed in Table 1. We can observe that the proposed SADL achieves $78.4 \%$ accuracy, $1.2 \%$ improvement over the second best method, LDL. Especially, the proposed SADL has over 5\% improvement 


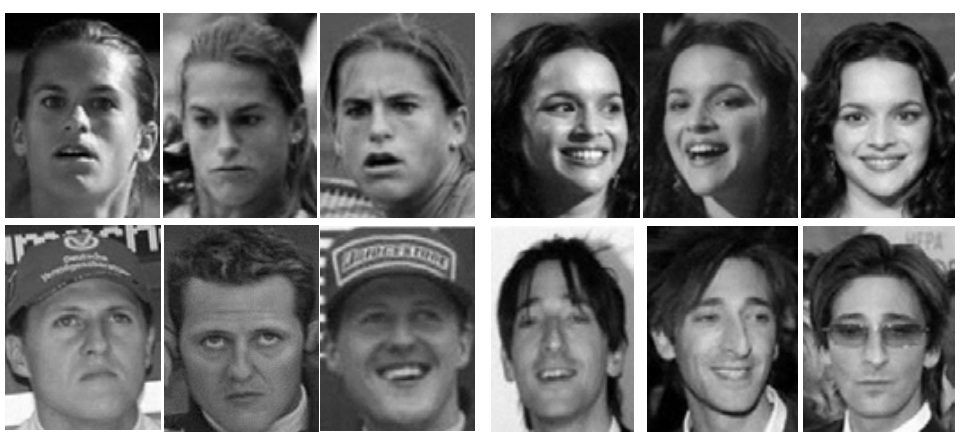

Figure 4: Some face images of LFWa.

Table 1: The Face Recognition Results (\%) of Different Methods on The LFW Database

\begin{tabular}{llll}
\hline Methods & Accuracy & Methods & Accuracy \\
\hline SRC-GC(LC) & $72.7(51.8)$ & COPAR & 72.6 \\
\hline DLSI & 73.8 & JDL & 72.8 \\
\hline DKSVD & 65.9 & FDDL & 74.8 \\
\hline LCKSVD & 66.0 & LDL-GC(LC) & $77.2(52.1)$ \\
\hline SVM & 63.0 & SADL-GC(LC) & $\mathbf{7 8 . 4 ( 5 3 . 8 )}$ \\
\hline
\end{tabular}

compared to the hybrid dictionary learning methods, such as COPAR and JDL, and more than $12 \%$ improvement compared to the shared dictionary learning methods, such as DKSVD and LCKSVD.

Based on the learnt binary matrix, we can not only capture the relationships between dictionary atoms and class labels, but also the relationships between class labels, which is shown as Fig. 1 .

\subsection{Gender Classification}

As in [23], we chose 14 images with illumination and expression changes per person (non-occluded) from the AR face database [30], which consists of 50 males and 50 females, to conduct experiments of gender classification. Some samples of male and female are shown in Fig. 5. Images of the first 25 males and 25 females were used for training and the remaining for testing. Dimension of each image was reduced to 300 by PCA and the class-specific dictionary size was initially set to 250 as [6]. The performance of differen$\mathrm{t}$ methods is shown in Table 2. We can see that the proposed SADL can 


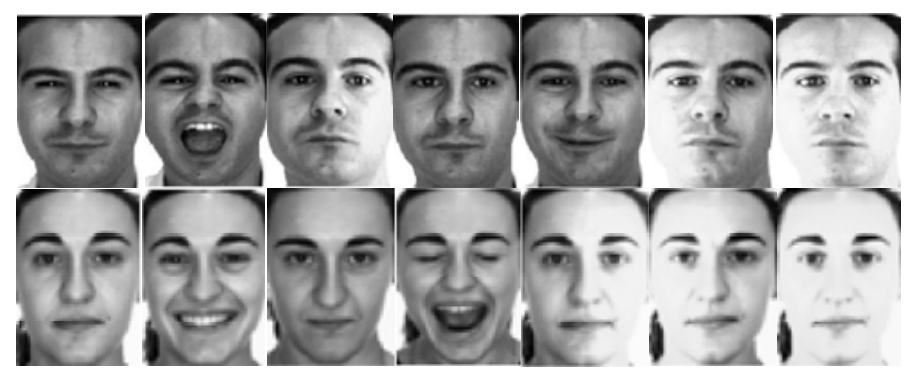

Figure 5: Some samples of males and females from AR database.

Table 2: The Gender Classification Rate(\%) on The AR Database With 250 Initialized Class-specific Dictionary Atoms Per Class

\begin{tabular}{llll}
\hline Methods & Accuracy & Methods & Accuracy \\
\hline SRC-LC(GC) & $93.0(91.5)$ & COPAR & 93.4 \\
\hline DLSI & 94.0 & JDL & 92.6 \\
\hline DKSVD & 86.1 & FDDL & 94.3 \\
\hline LCKSVD & 86.8 & LDL-LC(GC) & $95.3(94.8)$ \\
\hline SVM & 92.4 & SADL-LC(GC) & $\mathbf{9 5 . 7}(94.8)$ \\
\hline
\end{tabular}

learn a more compact dictionary by assigning the labels of dictionary atoms adaptively. From Table 2, we can see that the proposed SADL achieves the highest accuracy and has $0.4 \%$ improvement over LDL with smaller dictionary size.

Since class-specific dictionary usually has a big size, here we reduce the number of class-specific dictionary atoms per class from 250 to 25, and compare SADL with LDL, JDL, COPAR, DLSI and FDDL. The performance is reported in Table 3. It can be seen that the accuracies of all methods except SADL drop a little, however, SADL achieves $96.8 \%$ accuracy, $1.8 \%$ improvement over the second best method, LDL. In the case that only a small number of dictionary atoms but sufficient training samples, the relationship learnt by SADL is more suitable and flexibly to represent the data of different classes.

\subsection{Action Classification}

The UCF sports action database [31] contains 140 videos collected from various broadcast sports channels(e.g., BBC and ESPN), and covers 10 sport action classes: driving, golfing, kicking, lifting, horse riding, running, 
Table 3: The Gender Classification Rate (\%) on The AR Database With 25 Initialized Class-specific Dictionary Atoms Per Class

\begin{tabular}{c|c|c|c|c|c}
\hline DLSI & COPAR & JDL & FDDL & LDL-LC(GC) & SADL-LC(GC) \\
\hline 93.7 & 93 & 91.0 & 93.7 & $95.0(92.4)$ & $\mathbf{9 6 . 8}(95.8)$ \\
\hline
\end{tabular}

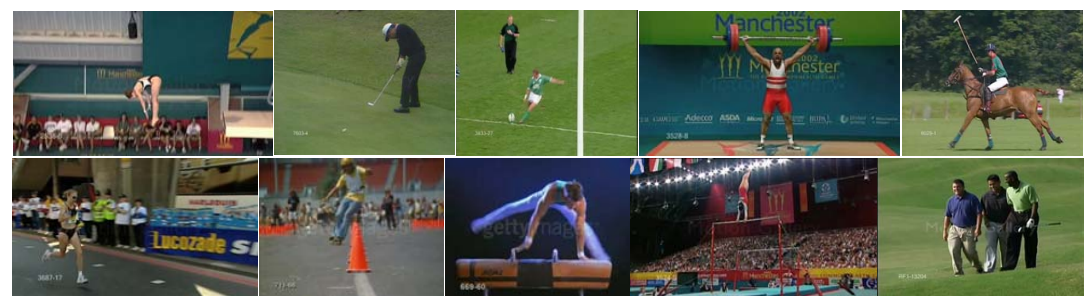

Figure 6: Some video frames of the UCF sports action database.

skateboarding, swinging(pommel horse and floor), swinging(high bar) and walking, some of which are shown in Fig. 6. The action bank features provided by [32] were adopted in the experiment. The dimension was reduced to 100 via PCA and dictionary size was the sum of all training samples.

Similarly with the experiment setting in [13, 24], we evaluated our method via five-fold cross validation and the recognition rates are listed in Table 4. Action bank feature with SVM classifier [32] is also reported. We can observe that the accuracy of proposed SADL is the same with LDL and higher than other dictionary learning methods.

\section{Conclusions}

In this paper, we presented a novel method, SADL, that learns a structure adaptive dictionary and a binary matrix jointly for sparse representation

Table 4: Classification Rate (\%) on the UCF Sports Action Database

\begin{tabular}{llll}
\hline Methods & Accuracy & Methods & Accuracy \\
\hline Sadanand & 90.7 & COPAR & 90.7 \\
\hline SRC-GC(LC) & $92.9(92.1)$ & JDL & 90.0 \\
\hline DLSI & 92.1 & FDDL & 93.6 \\
\hline DKSVD & 88.1 & LDL-GC(LC) & $\mathbf{9 5 . 0}(90.7)$ \\
\hline LCKSVD & 91.2 & SADL-GC(LC) & $\mathbf{9 5 . 0}(92.9)$ \\
\hline
\end{tabular}


based classification. SADL can adaptively learn the label and the property of each dictionary atom. Based on the learnt binary matrix, we can construct the graph structure between class labels. Meanwhile, we adopt Fisher criterion and weighted dictionary coherence term to learn a more discriminative dictionary. We performed experiments on face, gender and action classification, which demonstrated the effectiveness of SADL. In the future, we will work on how to characterize the coherence between dictionary atoms more accurately and reduce the correlations between dictionary atoms.

\section{Acknowledgments}

The authors would like to thank the editor and the anonymous reviewers for their critical and constructive comments and suggestions. This work was partially supported by the National Science Fund for Distinguished Young Scholars under Grant Nos. 61125305, 91420201 6147218761233011 and 61373063, the Key Project of Chinese Ministry of Education under Grant No. 313030, the 973 Program No. 2014CB349303, Fundamental Research Funds for the Central Universities No. 30920140121005, and Program for Changjiang Scholars and Innovative Research Team in University No. IRT13072.

\section{References}

[1] M. E. Julien Mairal, G. Sapiro, Sparse representation for color image restoration, IEEE Trans on Image Processing 17 (1) (2008) 53-69.

[2] J. Wright, A. Y. Yang, A. Ganesh, Y. Ma, Robust face recognition via sparse representation, Pattern Analysis and Machine Intelligence, IEEE Transactions on 31 (2) (2009) 210-227.

[3] B. A. Olshausen, D. J. Field, Emergence of simple-cell receptive field properties by learning a sparse code for natural images, Letters to Nature 381 (13).

[4] M. Yang, L. Zhang, J. Yang, D. Zhang, Metaface learning for sparse representation based face recognition, in: ICIP, 2010.

[5] J. Marial, F. Bach, J. Ponce, Task-driven dictionary learning, Pattern Analysis and Machine Intelligence, IEEE Transactions on 34 (4) (2012) 791-804. 
[6] Q. Zhang, B. Li, Discriminative k-svd for dictionary learning in face recognition, in: CVPR, 2010.

[7] M. Yang, P. Zhu, F. Liu, L. Shen, Joint representation and pattern learning for robust face recognition, Neurocomputing (168) (2015) 7080 .

[8] S. Kong, D. Wang, Learning inter-related visual dictionary for object recognition, in: ECCV, 2012.

[9] M. Yang, L. Zhang, X. Feng, D. Zhang, Sparse representation based fisher discrimination dictionary learning for image classification, Int $\mathrm{J}$ Comput Vis 109 (2014) 209-232.

[10] B. F. P. J. S. G. . Z. Mairal, J., Learning discriminative dictionaries for local image analysis, in: CVPR, 2008.

[11] . V. S. Pham, D., Joint learning and dictionary construction for pattern recognition, in: CVPR, 2008.

[12] J. Marial, F. Bach, J. Ponce, G. Sapiro, A. Zesserman, Supervised dictionary learning, in: NIPS, 2008.

[13] Z. Jiang, Z. Lin, L. S. Davis, Label consistent k-svd: Learning a discriminative dictionary for recognition, Pattern Analysis and Machine Intelligence, IEEE Transactions on 35 (11) (2013) 2651-2664.

[14] Y. Song, W. Can, Z. He, Robust iris recognition using sparse error correction model and discriminative dictionary learning, Neurocomputing (137) (2014) 198-204.

[15] H. Zheng, D. Tao, Discriminative dictionary learning via fisher discriminative k-svd algorithm, Neurocomputing (162) (2015) 9-15.

[16] J. Dong, C. Sun, W. Yang, A supervised dictionary learning and discriminative weighting model for action recognition, Neurocomputing (158) (2015) 246-256.

[17] P. Sprechmann, G. Sapiro, Dictionary learning and sparse coding for unsupervised clustering, in: Proceedings of the International Conference on Acoustics Speech and Signal Processing, 2010. 
[18] I. Ramirez, P. Sprechmann, G. Sapiro, Classification and clustering via dictionary learning with structured incoherence and shared features, in: CVPR, 2010.

[19] W. H. Haoran Wang, Chunfeng Yuan, C. Sun, Supervised class-specific dictionary learning for sparse modeling in action recognition, Pattern Recognition 45 (11) (2012) 3902-3911.

[20] M. Yang, L. Zhang, X. Feng, D. Zhang, Fisher discrimination dictionary learning for sparse representation, in: ICCV, 2011.

[21] A. Castrodad, G. Sapiro, Sparse modeling of human actions from motion imagery, International Journal of Computer Vision 100 (2012) 1-15.

[22] D. Meng, Q. Zhao, Y. Leung, Z. Xu, Learning dictionary from signals under global sparsity constraint, Neurocomputing (119) (2013) 308-318.

[23] N. Zhou, Y. Shen, J. Peng, J. Fan, Learning inter-related visual dictionary for object recognition, in: CVPR, 2012.

[24] M. Yang, D. Dai, L. Shen, L. V. Gool, Latent dictionary learning for sparse representation based classification, in: CVPR, 2014.

[25] Y. Karklin, M. S.Lewicki, A hierarchical bayesian model for learning nonlinear statistical regularities in nonstationary natural signals, Neuralcomputation 17 (2) (2005) 397-423.

[26] B. J. Culpepper, J. Sohl-Dichstein, B. A. Olshausen, Building a better probabilistic model of images by factorization, in: ICCV, 2011.

[27] L. Rosasco, A. Verri, M. Santoro, S. Mosci, S. Villa, Iterative projection methods for structured sparsely regularization, Tech. rep., MIT Technical Reports (2009).

[28] J. Mairal, F. Bach, J. Ponce, G. Sapiro, Online learning for matrix factorization and sparse coding, Journal of Machine Learning Research (11) (2010) 19-60.

[29] L. Wolf, T. Hassner, Y. Taigman, Similarity scores based on background samples, in: ACCV, 2009. 
[30] A. Martinez, R. Benavente, The ar face database, Tech. rep., CVC Tech. Report (1998).

[31] M. Rodriguez, J. Ahmed, M. Shah, A spatio-temporal maximum average correlation height filter for action recognition, in: CVPR, 2008.

[32] S. Sadanand, J. Corso, Action bank: A high-level representation of activity in video, in: CVPR, 2012. 